\title{
Designing Experiments in Cycles
}

DOI:

10.1016/j.chemolab.2018.11.009

\section{Document Version}

Accepted author manuscript

Link to publication record in Manchester Research Explorer

\section{Citation for published version (APA):}

Donev, A., \& Sammut-Powell, C. (2018). Designing Experiments in Cycles. Chemometrics and Intelligent Laboratory Systems. https://doi.org/10.1016/j.chemolab.2018.11.009

\section{Published in:}

Chemometrics and Intelligent Laboratory Systems

\section{Citing this paper}

Please note that where the full-text provided on Manchester Research Explorer is the Author Accepted Manuscript or Proof version this may differ from the final Published version. If citing, it is advised that you check and use the publisher's definitive version.

\section{General rights}

Copyright and moral rights for the publications made accessible in the Research Explorer are retained by the authors and/or other copyright owners and it is a condition of accessing publications that users recognise and abide by the legal requirements associated with these rights.

\section{Takedown policy}

If you believe that this document breaches copyright please refer to the University of Manchester's Takedown Procedures [http://man.ac.uk/04Y6Bo] or contact uml.scholarlycommunications@manchester.ac.uk providing relevant details, so we can investigate your claim.

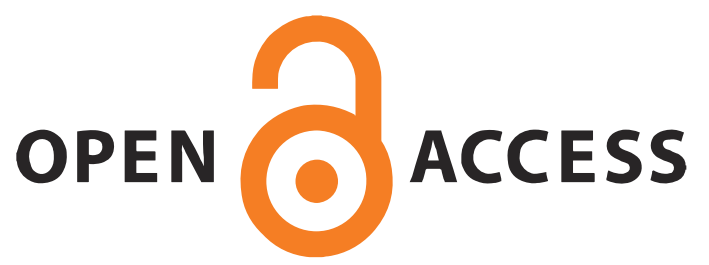




\section{Accepted Manuscript}

Designing experiments in cycles

Alexander N. Donev, Camilla Sammut-Powell

PII: S0169-7439(18)30540-9

DOI: $\quad$ https://doi.org/10.1016/j.chemolab.2018.11.009

Reference: $\quad$ CHEMOM 3711

To appear in: Chemometrics and Intelligent Laboratory Systems

Received Date: 20 September 2018

Revised Date: 5 November 2018

Accepted Date: 13 November 2018

Please cite this article as: A.N. Donev, C. Sammut-Powell, Designing experiments in cycles, Chemometrics and Intelligent Laboratory Systems (2018), doi: https://doi.org/10.1016/ j.chemolab.2018.11.009.

This is a PDF file of an unedited manuscript that has been accepted for publication. As a service to our customers we are providing this early version of the manuscript. The manuscript will undergo copyediting, typesetting, and review of the resulting proof before it is published in its final form. Please note that during the production process errors may be discovered which could affect the content, and all legal disclaimers that apply to the journal pertain. 


\title{
Designing Experiments in Cycles
}

\author{
Alexander N. Donev and Camilla Sammut-Powell \\ University of Manchester, Oxford Road, Manchester, M13 9PL, UK
}

Abstract: Experimental observations are frequently collected sequentially, allowing for lurking variables to affect the response and cause a trend in the results. Often the order in which the observations are collected is randomised to minimise the impact. However, when information about the behaviour of the trends has been found in previous experiments, it is possible to use this knowledge to obtain better designs that ensure that the studied relationship and the effect of the trend are estimated accurately. We consider cases where the cyclic nature of the experiments allows for obtaining such information prior to the main study. We propose a general methodology for designing such experiments, based on the framework of generalised linear models. The proposed methodology considers a larger class of problems than previously researched, allowing for the effect of a time trend on both the mean and variance of the response.

Key words and phrases: compound design criterion, D-optimality, design construction, generalised linear models, multivariate response 


\section{Introduction}

Blocking is an elegant technique used when designing experiments. Given a response of interest, it aims to improve the precision of the parameter estimates of a statistical model. The most common way to implement it is by allocating the experimental units to blocks so that the within block variability is expected to be smaller than the variability across the blocks. The between block variability on the response explains some of the total variation, rather than inflating the residual variance. This has played a fundamental role in experimental research for decades.

There are experiments where the experimental units of each block have to be used sequentially. In these cases, time-related factors may affect them and thus destroy their homogeneity. Such an effect may be possible to describe as a trend that affects the response measurements in all blocks consistently, potentially to both their means and variances. A typical example is provided in Section 2 where a device is calibrated at the start of each day and then used to collect groups of measurements of the response for different values of the explanatory variables. The device gradually changed its performance over the day, causing a trend in the measurements hence the need for a calibration each day. The trend effect has a cyclic manner as it is repeated each day. Observations in a block correspond to all observations taken on a particular day. Due to their specific nature, we refer to the blocks as cycles. Such cyclic changes in the experimental condition often exist in experimental research in chemistry, biochemistry, engineering, etc.

The need to take into account time trends in experiments is well recognised. Cox (1951) introduced this problem by giving an example where the properties of wool fibre were affected not only by treatments of interest but also by the time of their use due to the ageing of the wool. The most common approach to address such problems is to randomise 
the order in which the observations are collected. This usually reduces the probability of obtaining biased results. The use of trend-free designs has been recommended when the form of the trend is known (e.g. linear or quadratic). For example, Atkinson and Donev (1996) consider the case when a trend affects the mean of a response in the presence of homogeneous, additive normally distributed observations. They construct $D$-optimal designs considering the trend defining parameters as nuisance parameters. The trendfree designs that they obtained were linear in the model parameters and the estimates of these were unaffected by the effect of the trend. Atkinson and Donev (1996) showed that there were situations where trend-free designs could not be obtained and defined a criterion that could be used to measure the robustness of a design to a particular type of trend. However, their methodology has important limitations and cannot be used when the response is multivariate (as different trends could affect the responses), the experimental errors have heterogeneous variance or may be dependent, nor when the response is not normally distributed. Subsequent research broadens the situations where trend robust designs can be used but it still has similar shortcomings. Further details are provided in Section 3, where we focus on the approach proposed by Atkinson and Donev (1996). In Section 4 we extend their idea to cover a much wider range of trend-related problems. Motivated by the example given in Section 2, we are interested in the development of methodology for designing experiments where the response can be multivariate, a trend can affect both the mean and the variance of the responses and the experimental errors associated with the responses have different distributions.

The first key step in this process is to allow for more complex trend-related effects than previously considered in the statistical model of interest. This is achieved by formulating the model of interest as a generalised linear model (GLM) that accommodates 
the effect of the trend on the mean response in the linear predictor. If the mean and the variance of the response are unrelated, a parametric relationship can be specified to capture the effect of the trend on the variance of the response.

GLMs are well studied and their estimation can be easily carried out using all main statistical packages. Estimating the effect of the trend is done at same time as estimating the effect of all other explanatory variables. This is in sharp contrast to the analysis of data collected with the use of trend-free designs constructed in the past, where the effect of the trend may not be even possible to estimate. Our approach is quite general allowing for the distribution of the response to be any member of the exponential family of distributions (EFD). Hence, our approach benefits from the existing methodology for designing experiments for estimating GLMs. For a review of this methodology see Atkinson et al. (2007) and Stufken and Yang (2012).

A common difficulty in designing experiments for estimating GLMs is that finding a solution requires knowledge about the values of the model parameters that is the aim of the study to estimate. This problem is usually addressed by using the best available estimates, or their distribution. Inevitably, the required design optimality is satisfied only approximately.

When the experiments are carried out in cycles, the parameters can be estimated initially in a small validation study, or be known from previous similar experiments. Therefore our approach finds reliably efficient experimental designs with block cycles.

Finally, constructing a design for several responses that is robust to trend effects can be done with carefully defined compound criterion of optimality. In Section 5 we illustrate the new methodology by returning to the example given in Section 2. The paper concludes with a discussion of the merits and limitations of our approach. 


\section{Supplementary Material}

An $R$ program used to generate the three experimental designs discussed in the paper, or similar designs, is provided as supplementary material. It is made available on the journals' and the authors'webpages.

\section{Example: Hot Pressing Process}

Various products (e.g., electrical fitting products such as light bulb holders, switch covers, circuit boards and plugs) are manufactured by hot pressing. At the start of each production run, a quantity of moulding powder and resin is loaded into a hopper above the press. The base of the hopper has a screw feeder into an automated weighing system, which weighs out a set amount for each product. This is dropped into the hot die. The die closes and pressure is applied until the resin cures. The press then opens and the product is ejected. The process is automatic.

Screw conveyors work very well for liquids and slurry but tend to "tunnel" and "bridge" with dry powders, which stops them from conveying. The usual solution is to have a paddle or rotor that slowly moves the bulk of the powder in the hopper to keep the screw feeder running properly. This slow motion combined with the vibration from the press generates segregation in the moulding powder. Therefore, the particle size distribution of the powder samples used to manufacture products changes over time and causes a trend in the properties of the manufactured products.

At the start of each day the device is calibrated and the moulding powder is homogeneous. As production continuous, the hopper paddle and vibration tend to segregate the moulding powder with the finer particles accumulating at the bottom of the hopper at 
the screw feeder and the bigger particles percolating up to the top. The smaller particles compact easier than the large particles. Hence, product density increases during the first part of the production cycle, then decreases in the second part of the cycle as the smaller particles are used and the particle size distribution of the powder drifts upwards. The easily compacted smaller particles give a much more consistent product than the more granular material at the end of the production cycle, and even when compared with the powder used right at the start of the cycle. This causes the properties of the products to change dependent on the time it was manufactured.

Therefore, the proportion of defective products depends on both the operating conditions and the time trend caused by the changing particle size distribution of the moulding powder. The proportion of defective items $\left(Y_{1}\right)$ was of interest and had a binomial distribution. The second response $\left(Y_{2}\right)$ was the strength of the product. It was measured by weighing the products. Previous similar experiments suggested that the distribution of the strength was approximately normal, and its variance was proportional to its mean. Hence, the time trend affected both the mean and the variance of the response each day in a similar way, and measurements taken on a particular day were considered a block cycle. Previous experience suggested that the measurements of both responses were independent.

The process was affected by four variables: hopper paddle speed $\left(x_{1}\right)$, press temperature $\left(x_{2}\right)$, press time $\left(x_{3}\right)$, and auto-weigher setting $\left(x_{4}\right)$. Their optimum values were found experimentally for each type of product. In an experiment both $Y_{1}$ and $Y_{2}$ were measured for each produced item and the time $\left(x_{5}\right)$ of taking each measurement was also recorded.

An experimental design with $n=25$ different settings of the process variables was 
used. Five different settings were tested sequentially at equal intervals of time on each of the days of a 5-day working week. Hence, there were 5 cycles. The equipment was calibrated at the start of each cycle. Fresh powder was used each day. Samples of 25 items were obtained for each of the settings and the responses were recorded (i.e., 625 measurements were collected in total). The 25 items taken for each setting were affected equally by the time trend as these items were made in a quick succession. Therefore the trend effects on the responses was determined by the time in the day at which the settings were tested.

Generalised linear models were fitted for each of the responses, both with canonical link functions. Stepwise regression was used to choose the required predictors from the terms of a second order polynomial in the process variables and the time in the day when the observations were taken, excluding all interactions between $x_{5}$ and the remaining variables. Different regressors were needed in the linear predictors for the models of the two response variables. The response $Y_{1}$ had 13 regressors (intercept, $x_{1}, x_{2}$, $\left.x_{3}, x_{4}, x_{5}, x_{1} x_{3}, x_{1} x_{4}, x_{2} x_{3}, x_{2} x_{4}, x_{3} x_{4}, x_{4}^{2}, x_{5}^{2}\right)$, with corresponding parameter estimates $1.326,0.327,0.308,-0.511,-1.069,0.964,1.033,-1.240,-0.436,0.314,0.389,0.352,-0.298$. The response $Y_{2}$ had 15 regressors (intercept, $x_{1}, x_{2}, x_{3}, x_{4}, x_{5}, x_{1} x_{2}, x_{1} x_{3}, x_{1} x_{4}, x_{2} x_{3}$, $\left.x_{2} x_{4}, x_{3} x_{4}, x_{2}^{2}, x_{4}^{2}, x_{5}^{2}\right)$, with corresponding parameters estimates 4.029, 0.170, 0.397, -0.256 , $0.300,0.345,-0.501,0.357,-0.678,0.162,-0.253,-0.344,0.212,-0.433,-0.306$. Remember that $x_{5}$ is the scaled time of taking the observations. 


\section{Background}

Early work on design of experiments in the presence of time trends focussed on experiments to compare several treatments. Designs for collecting data to estimate treatment effects identical to those that would have been obtained if the observations were taken at the same time, or there were no time trend, are considered trend free. While the form of the time trend is assumed to be known (e.g., linear or quadratic) to construct such designs, no information about the model parameters is required. For example, Cheng (1990) provides an extensive review of research devoted to trend free ordering of treatments compared experimentally.

Atkinson and Donev (1996) consider the case when a trend affects the mean in a response surface study where the relationship between the response $Y$ and the explanatory variables can be described with the model

$$
\mathbf{Y}=\mathbf{F} \boldsymbol{\beta}+\boldsymbol{\varepsilon}
$$

where $\mathbf{Y}$ is an $n \times 1$ vector of the observations, $\mathbf{F}$ is an $n \times p$ design matrix, and $\boldsymbol{\beta}$ is an $p \times 1$ vector of the model parameters. The experimental errors of the observations, gathered in the $n \times 1$ vector $\varepsilon$, are assumed to be additive, independent and have a normal distribution with zero mean and homogenous variance $\sigma^{2}$. That is, $\boldsymbol{\varepsilon} \sim \mathrm{N}_{n}(\mathbf{0}, \boldsymbol{\Sigma})$, where $\boldsymbol{\Sigma}=\sigma^{2} \mathbf{I}$ and $\mathbf{0}$ is an $n \times 1$ vector of zeros. High precision of estimating the model parameters is important and therefore $D$-optimality is beneficial as a design criterion. This criterion requires maximising the determinant of the information matrix, $\left|\mathbf{F}^{\mathrm{T}} \mathbf{F}\right|$, which minimises the volume of the confidence region for the model parameters.

We provide further details of the approach of Atkinson and Donev (1996) as we build 
on it in Section 4. The effect of the time trend on the mean may be parametrised as $\sum_{j=1}^{s} \delta_{j} g_{j}\left(t_{i}\right)$, where $t_{i}(i=1, \ldots, n)$ is the time of collecting the $i$ th observation, and $g_{j}\left(t_{i}\right)$ and $\delta_{j}(j=1,2, \ldots, s)$ are functions and the corresponding parameters describing the time trend. As a result of the time trend, the underlying model becomes

$$
E[\mathbf{Y}]=\mathbf{F} \boldsymbol{\beta}+\mathbf{Z} \boldsymbol{\delta}=\mathbf{X} \boldsymbol{\lambda},
$$

where $\mathbf{F}$ is the $n \times p$ design matrix, $\mathbf{Z}$ is the $n \times s$ matrix whose $i$ th row contains the values $g_{j}\left(t_{i}\right), j=1,2, \ldots, s$, and $\boldsymbol{\delta}$ is the $s \times 1$ vector of the parameters related to the time trend. Also, $\mathbf{X}$ is the $n \times(p+s)$ matrix combining the matrices $\mathbf{F}$ and $\mathbf{Z}$, and similarly the $(p+s) \times 1$ vector $\boldsymbol{\lambda}$ combines the vectors $\boldsymbol{\beta}$ and $\boldsymbol{\delta}$. The $\mathrm{D}_{s}$-optimum design for model (3.2), which treats the parameters $\boldsymbol{\delta}$ as nuisance parameters, maximises $\left|\mathbf{X}^{\mathrm{T}} \mathbf{X}\right| /\left|\mathbf{Z}^{\mathrm{T}} \mathbf{Z}\right|$. Certainly this is a constrained optimisation problem where $\left|\mathbf{Z}^{\mathrm{T}} \mathbf{Z}\right|$ should not be allowed to reach zero. This is usually ensured by appropriately predetermining the levels of the time variable. Constrained optimisation design problems have been considered by Cook and Thibodeau (1980).

Atkinson and Donev (1996) define the criterion

$$
T F=\left(\frac{\left|\mathbf{X}^{\mathrm{T}} \mathbf{X}\right| /\left|\mathbf{Z}^{\mathrm{T}} \mathbf{Z}\right|}{\left|\mathbf{F}^{* \mathrm{~T}} \mathbf{F}^{*}\right|}\right)^{1 / p},
$$

where $\mathbf{F}^{*}$ is the design matrix of the $D$-optimum design in the absence of trend. The maximum value of $T F$ is 1 , attained when the design is perfectly balanced for the trend. In this case, the least squares estimates of the parameters of interest $\hat{\boldsymbol{\beta}}$ will be the same as in the case when all the observations were collected at the same time or there was no 
time trend. This criterion is used to construct designs that are trend free for a specified time trend.

The case when a time trend affects the results was studied by many researchers. For example, Tack and Vandebroek (2002) and Tack and Vandebroek (2003) consider the case when estimating a mixed effect model is required, while Tack and Vandebroek (2004a) and Tack and Vandebroek (2004b) study the impact of the order of the observations to total experimental cost. Jones and Goos (2011), Harman and Sagnol (2015) and Jones and Goos (2015) present the problem as an example of an experimental situation where uncontrollable covariates affect the response. Similar is Wang and Ai (2016) where the response has heterogeneous variance. Most of the related research in the past has been focused on $D$-optimality of the designs, but Rosa and Harman (2016) and Wang and Ai (2016) consider a wider range of optimality criteria.

However, designing experiments in the presence of a trend when the response is multivariate, and hence different trends can affect the responses, has not been studied in the past. Furthermore, each response may have non-gaussian distribution, hence the time trend will affect both their mean and variance. In Section 4 we show how such an effect can be accommodated in a linear regression model.

\section{GLM models with time trend}

GLMs provide a natural framework to describe data when there is a time trend that affects the mean and/or the variance of the response. First we summarise the main features of a GLM and then describe how they can be used to accommodate various possible effects of a time trend. 
GLMs were introduced by Nelder and Wedderburn (1972). A GLM is defined by three aspects:

- the distribution of the response, which belongs to the exponential family,

- the linear predictor, $\boldsymbol{\eta}=\mathbf{F} \boldsymbol{\beta}$, and

- the link function, $g$, such that $E[K(\mathbf{Y})]=\boldsymbol{\mu}=g^{-1}(\boldsymbol{\eta})$, where $\mathbf{Y}$ is the vector of observed response values, $\boldsymbol{\mu}$ is the mean of $K(\mathbf{Y})$ and $K(\cdot)$ is a known function of Y.

The general form of the probability density function of a distribution from the exponential family of distributions (Pitman, 1936) for the $i$ th observation is given by

$$
f_{Y_{i}}\left(y_{i} \mid \theta_{i}, \phi_{i}\right)=h\left(y_{i}, \phi_{i}\right) \exp \left[\frac{B\left(\theta_{i}\right) K\left(y_{i}\right)-A\left(\theta_{i}\right)}{d\left(\phi_{i}\right)}\right]
$$

where $\theta_{i}$ is the natural parameter and the observations are assumed independent. Also $E\left[K\left(Y_{i}\right)\right]=A^{\prime}\left(\theta_{i}\right) / B^{\prime}\left(\theta_{i}\right)$ and

$$
\operatorname{var}\left(K\left(Y_{i}\right)\right)=\frac{d\left(\phi_{i}\right)}{B^{\prime}\left(\theta_{i}\right)^{3}}\left(A^{\prime \prime}\left(\theta_{i}\right) B^{\prime}\left(\theta_{i}\right)-B^{\prime \prime}\left(\theta_{i}\right) A^{\prime}\left(\theta_{i}\right)\right)
$$

Hence, the variance consists of a scale function, $d\left(\phi_{i}\right)$, and a variance function,

$$
V\left(\theta_{i}\right)=\frac{A^{\prime \prime}\left(\theta_{i}\right) B^{\prime}\left(\theta_{i}\right)-B^{\prime \prime}\left(\theta_{i}\right) A^{\prime}\left(\theta_{i}\right)}{B^{\prime}\left(\theta_{i}\right)^{3}} .
$$

The variance function is often written in terms of $\mu_{i}=E\left[K\left(Y_{i}\right)\right]$ to describe the relationship between the mean and variance of $K\left(Y_{i}\right)$. 
Because the observations are independent, a GLM with design matrix $\mathbf{F}$ has an information matrix that can be written as a sum of the information matrices for the individual observations

$$
\mathbf{F}^{\mathrm{T}} \mathbf{W} \mathbf{F}=\sum_{i=1}^{n} w_{i} \mathbf{f}_{i} \mathbf{f}_{i}^{\mathrm{T}},
$$

where $\mathbf{f}_{i}^{\mathrm{T}}$ is the $i$ th row of the $\mathbf{F}$ matrix, and $\mathbf{W}$ is a diagonal matrix. The $i$ th diagonal element of $\mathbf{W}$ is

$$
w_{i}=\frac{1}{\operatorname{var}\left(K\left(y_{i}\right)\right)}\left(\frac{d \mu_{i}}{d \eta_{i}}\right)^{2}
$$

Similarly to Atkinson and Donev (1996) we accommodate the effect of a trend in the model. However, using the GLM framework allows us to cover a much wider range of possible time trend effects. Here are several possible cases, each requiring a different presentation in the model.

- A time trend affects the mean only. In this case $\eta_{i}$ has to accommodate the effect of the trend and $V\left(\theta_{i}\right)=1$.

- A time trend affects only the variance. Then, $\phi_{i}$ has to accommodate the effect of the trend.

- A time trend affects both the mean and variance. This can occur through $\eta_{i}$ alone, or a combination of $\eta_{i}$ and $\phi_{i}$.

If there is a trend in the linear predictor, the extended design matrix is $\mathbf{X}=\left[\begin{array}{ll}\mathbf{F} & \mathbf{Z}\end{array}\right]$, where $\mathbf{F}$ is the design matrix for the variables of interest and $\mathbf{Z}$ is the design matrix for the trend. This gives a linear predictor of the same form as (3.2), i.e.

$$
\boldsymbol{\eta}=\mathbf{F} \boldsymbol{\beta}+\mathrm{Z} \boldsymbol{\delta}=\mathrm{X} \boldsymbol{\lambda}
$$


When the trend affects only the variance, e.g., when the response has a normal distribution with heterogeneous variance, $\phi_{i}$ takes a form that represents this effect. For example, in many situations trends in the variance of the response can be represented by

$$
a\left(\phi_{i}\right)=\sigma^{2} \sum_{j=1}^{k} \gamma_{j} u_{j}\left(\mathbf{x}_{i}\right)
$$

where $\mathbf{x}_{i}$ is the vector of the values of the explanatory variables for the $i$ th observation, and $u_{j}\left(\mathbf{x}_{i},\right)$ and $\gamma_{j}, j=1,2, \ldots, k$, are the functions and their corresponding parameters describing the variance. This and alternative possibilities for presenting the heterogeneity of normally distributed errors have been discussed in the literature, for example by Atkinson and Cook (1995).

If the mean and the variance of the response are related (e.g., when the distribution is binomial or Poisson) such a trend affects the variance of the response accordingly. We now consider several typical cases of GLMs that accommodate time trends.

\section{Case 1. Normally distributed response, additive errors with homogeneous}

variance. When the errors have a normal distribution with zero mean and homogeneous variance, model (3.2) accommodates the effect of a trend on the mean $\mu_{i}=\eta_{i}=$ $\mathbf{f}_{i}^{\mathrm{T}} \boldsymbol{\beta}+\mathbf{z}_{i}^{\mathrm{T}} \boldsymbol{\delta}$, where $\mathbf{z}_{i}^{\mathrm{T}}$ is the $i$ th row of the $\mathbf{Z}$ matrix. Also, $d\left(\phi_{i}\right)=\sigma^{2}, V\left(\theta_{i}\right)=1, w_{i}=\sigma^{-2}$.

\section{Case 2. Normally distributed response, additive errors with heterogeneous}

variance. When the errors have a normal distribution with zero mean and the variance is dependent on factors $\mathbf{x}_{i}$, a trend affecting only the variance can be represented by a GLM with $\mu_{i}=\eta_{i}=\mathbf{f}_{i}^{\mathrm{T}} \boldsymbol{\beta}, d\left(\phi_{i}\right)=\sigma^{2} \sum_{j=1}^{k} \gamma_{j} u_{j}\left(\mathbf{x}_{i}\right), V\left(\theta_{i}\right)=1$ and $w_{i}=1 /\left(\sigma^{2} \sum_{j=1}^{k} \gamma_{j} u_{j}\left(\mathbf{x}_{i}\right)\right)$. 
Case 3. Normally distributed response, multiplicative errors. Certainly this case can be reduced to the earlier cases by taking the logarithms of the observations. Alternatively, the model can be written as

$$
\mathbf{Y}=(\mathbf{X} \boldsymbol{\lambda}) \circ \varepsilon
$$

where $\circ$ is the Hadamard product. For example, if the distribution of the errors is lognormal (i.e., $\left.\boldsymbol{\varepsilon} \sim \operatorname{LN}\left(\mathbf{0}, \sigma^{2} \mathbf{I}\right)\right)$ the observations follow a log-normal distribution. That is, $\mathbf{Y} \sim \mathrm{LN}\left(\log (\mathbf{X} \boldsymbol{\lambda}), \sigma^{2} \mathbf{I}\right)$, where $\mathbf{X}$ is defined by (4.7) and the link function here being exponential. The log-normal distribution cannot be written in a canonical form, since $K\left(y_{i}\right)=\log \left(y_{i}\right)$. A trend affecting both the mean and the variance of the response can be represented by a GLM with $d\left(\phi_{i}\right)=\sigma^{2}, V\left(\theta_{i}\right)=1$ and $w_{i}=\sigma^{-2} \eta_{i}^{-2}$.

Case 4. Binomial response. The distribution of the response in the $i$ th observation is binomial, i.e. $B\left(n_{i}, p_{i}\right)$, where the probability of "success" is $p_{i}$ in all $n_{i}$ trials and $\mu_{i}=n_{i} p_{i}$. A trend will affect both the mean and the variance of the response through the natural parameter since $d\left(\phi_{i}\right)=\phi_{i}=1$ by definition and $V\left(\theta_{i}\right)=n_{i} e^{\theta_{i}} /\left(1+e^{\theta_{i}}\right)$. If a logistic link function is used, then it is possible to define a GLM accommodating an effect of a trend with $\eta_{i}=\mathbf{f}_{i}^{\mathrm{T}} \boldsymbol{\beta}+\mathbf{z}_{i}^{\mathrm{T}} \boldsymbol{\delta}, g\left(\mu_{i}\right)=\log \left(\mu_{i} /\left(1-\mu_{i}\right)\right)=\eta_{i}$ and $w_{i}=\mu_{i} /\left(1-\mu_{i}\right)$. If a different link function is used, $w_{i}$ has to be changed accordingly.

Case 5. Poisson response. If the response has a Poisson distribution, the situation is similar to that in Case 4 as it is possible to define a GLM with $\eta_{i}=\mathbf{f}_{i}^{\mathrm{T}} \boldsymbol{\beta}+\mathbf{z}_{i}^{\mathrm{T}} \boldsymbol{\delta}$ 
and $d\left(\phi_{i}\right)=\phi_{i}=1$. However, $g\left(\mu_{i}\right)=\log \left(\mu_{i}\right)=\eta_{i}, a\left(\phi_{i}\right)=1, V\left(\theta_{i}\right)=e^{\theta_{i}}=\mu_{i}$ and $w_{i}=\mu_{i}=\exp \left(\eta_{i}\right)$

In Case 1 the time trend affects only the mean of the response; in Case 2 it affects only the response variance, while in Cases 3,4 and 5 both the mean and the variance are affected. Formulation of suitable GLMs for other problems can be made in a similar way. Once the suitable GLM is defined, the next stage is to construct the required experimental design.

\section{Design construction}

We are interested in designs that are $D$-optimum (i.e., designs that ensure high precision of the estimation of the model parameters). Using other criteria for design optimality could also be useful, such as criteria concerned with the precision of the predictions using the fitted model. Previous experience suggests that using $D$-optimum designs lead to finding models which are also good in this respect.

The $D$-optimum design in the presence of the trend maximises

$$
D=\left|\mathbf{X}^{T} \mathbf{W X}\right|
$$

where $\mathbf{W}$ is a diagonal matrix with elements given by (4.6). Thus, we are able to present the problem as one for designing experiments for estimating parameters of a GLM. Similarly to nonlinear estimation problems, the solution depends on the values of the unknown model parameters. In the absence of such information, the criterion of 
optimality cannot be calculated. Often the most likely values, or their distribution, are assumed to evaluate (5.9), even though the result is only approximate.

The search for an optimum design can be done in many ways. However, one should run the optimisation at least 100 times with different random seeds to start the process. We used the $\mathrm{R}$ program provided in the supplementary material, implementing the adjustment algorithm of Donev and Atkinson (1988). Given an unlimited amount of computational time, this algorithm will find a globally optimum which may or may not be unique. However, in practice one will have a fixed amount of time to find a solution. This is why in our computer program the search can be terminated at the time when no further wait for the solution is possible. The search may not have found a global optimum design, but unless it has run for a very short amount of time, the best available design is likely to be satisfactory for practical use.

Example: Hot Pressing Process (continued). The results of the study described earlier were used to construct an experimental design for a new product. The same design structure as described in the example in Section 2 was used. That is, the experiment was to be run on five days, testing five different settings on each day sequentially at times scaled as $-1,-0.5,0,0.5$ and 1 . The same explanatory and response variables were under consideration and the aim was to identify the best process settings which would minimise the defects and increase the strengths of the items. It was assumed that similar models to those obtained in the previous study would apply. Therefore, such models, and their estimated parameters, were used in the design construction, hence the design that was found was only approximately optimal.

When a design was considered, (5.9) was used to calculate the criteria values for 
models $Y_{1}$ and $Y_{2}, D_{1}$ and $D_{2}$, respectively. A compound design criterion of optimality for estimating both models was used for the design construction. It was defined as the product of the criteria values (i.e., $D_{c}=D_{1} * D_{2}$ ). This avoided the possibility that a design that could not be used to estimate either of the models was chosen. This criterion is based on the assumption of no correlation between both responses.

For illustration, the best design in 1000 searches, each using a different seed to find the starting design, is shown in Table 1. For example, the eighth row shows the process settings $x_{1}=-1, x_{2}=-1, x_{3}=-1, x_{4}=1$ that will be tested second $\left(x_{5}=-0.5\right)$ on the the third day $(\operatorname{Day}=3)$ of the week. For this design $D_{1}=3.651 * 10^{4}, D_{2}=6.058 * 10^{11}$ and $D_{c}=2.212 * 10^{16}$.

It was also interesting to construct optimum designs for the individual response variables and compare them with the chosen design. The best design for estimating the binomial response has $D_{1}=1.485 * 10^{5}$. Using this design would not allow for estimating the required model for the second response (i.e., $D_{2}=0$ ). The best design for estimating the heterogeneous normally distributed response has (5.9) $D_{2}=1.7 \times 10^{12}$. Also, $D_{1}=9.171 * 10^{3}$ and $D_{c}=9.239 * 10^{14}$ for this design. These two designs are given in Table 2 and Table 3 in the Appendix.

The three designs that were found are locally $D$-optimum, because the model parameter estimates obtained in the previous study were used to construct them. Therefore, the three designs are clearly only approximately $D$-optimum, because the true values of the parameters may be different. However, a design constructed this way is likely to be much better than one obtained by ignoring the impact of a trend on the results. 
Table 1: Approximately $D$-optimum design for estimating both the binomial and the normal heterogeneous responses, $Y_{1}$ and $Y_{2}$, respectively.

\begin{tabular}{crrrrr}
\hline Day & $x_{1}$ & $x_{2}$ & $x_{3}$ & $x_{4}$ & $x_{5}$ \\
\hline 1 & -1 & -1 & -1 & -1 & -1.0 \\
1 & 1 & 1 & -1 & -1 & -0.5 \\
1 & 1 & -1 & 1 & 0 & 0.0 \\
1 & -1 & -1 & 1 & -1 & 0.5 \\
1 & -1 & 1 & -1 & 1 & 1.0 \\
2 & -1 & 1 & 1 & -1 & -1.0 \\
2 & -1 & 0 & 1 & 1 & -0.5 \\
2 & -1 & -1 & 1 & 0 & 0.0 \\
2 & 1 & 0 & -1 & 1 & 0.5 \\
2 & 1 & -1 & 1 & 1 & 1.0 \\
3 & 1 & 1 & -1 & 0 & -1.0 \\
3 & -1 & -1 & -1 & 1 & -0.5 \\
3 & 1 & -1 & 1 & 1 & 0.0 \\
3 & -1 & 1 & 1 & 1 & 0.5 \\
3 & -1 & 0 & -1 & 0 & 1.0 \\
4 & 1 & -1 & 1 & -1 & -1.0 \\
4 & 1 & 1 & 1 & 1 & -0.5 \\
4 & -1 & 1 & -1 & -1 & 0.0 \\
4 & 1 & -1 & -1 & -1 & 0.5 \\
4 & 1 & 1 & 1 & 0 & 1.0 \\
5 & 1 & -1 & -1 & 1 & -1.0 \\
5 & -1 & 1 & -1 & 1 & -0.5 \\
5 & -1 & -1 & -1 & 1 & 0.0 \\
5 & 1 & 0 & 1 & -1 & 0.5 \\
5 & -1 & -1 & 1 & 1 & 1.0 \\
\hline
\end{tabular}




\section{Discussion}

Most of the previous research on experimental designs in the presence of a time trend focussed on finding designs orthogonal to the effects of trends. Such designs provide for analysing the data as if there were no trend and obtaining estimates of the model parameters which would be identical to those obtained in the absence of a trend. However, if the trend turns out to be different from the anticipated one, this will affect the statistical properties of the results (e.g., the estimated model can be biased and the estimate of the prediction variance can be wrong). If the effect of the trend is not estimated, the experimenter may not even be aware of the problems in the statistical analysis of the data. Furthermore, such designs are rarely available, particularly if a trend is found to affect the variance, or if the response is multivariate. It is important to recognise that our approach highly differs from the literature by considering the effect of the trend to be important and ensuring its best possible estimation. Previous research considered the trend a nuisance effect that was not of interest to estimate. In particular, the current methodology advises the use of designs orthogonal to the effects of the trend is rarely practical and highly limited. In many cases, as illustrated in the hot pressing example, information about the trend can provide useful guidance on how to design future experiments.

When constructing designs using information from previous similar experiments, it may be that multiple datasets are available. If so, such information will allow for the robustness of the best design to be studied by calculating the criterion value across the values of the model parameters obtained in those previous experiments.

On contrary, there may be also cases when data from previous experiments are not 
available. The cyclic nature of the described experiments makes it possible to conduct initially one or two experimental cycles in order to obtain the required information for the construction of the design for the main study. These small experiments have a similar role to screening and validation experiments commonly used in experimental research.

Our new approach facilitates the construction of experimental designs where the mean and/or the variance of the response are affected by a trend. Using the framework of GLMs to present the problem allows for constructing designs in cases when the response follows one of many possible distributions: a considerable improvement on existing methodology.

The main challenges of designing experiments when a trend is expected to affect the results arise from the dependence of the optimum design on the unknown model parameters. Here we reiterate some specific features of the design problem that need to be taken into account:

- Estimating the trend is important. Only when a design is trend free does the criterion (3.3) have a clear interpretation (i.e., when its value is 1). This only occurs for a small number of cases.

- Customarily, the robustness of experimental designs with respect to all assumptions made in relation to their construction needs to be studied before the designs are used in practice. The designs constructed using the method presented in this paper are not exceptions. For example, it is useful to study the performance of a chosen design where the form of the trend differs to that assumed in the construction of the design. In most cases, many alternative time trends can be estimated under the new methodology. This allows us to obtain informative results, even when the 
design may be suboptimal for the true form of the trend. This is a seemingly small but important advantage of our methodology over existing ones.

- In most experiments the times of collecting data and the number of the observations taken at each time point will be decided by the specific features of the experiment. However, if there is some flexibility in choosing the time of the measurements during each cycle, our approach can be extended to select the best time points with respect the chosen design criterion. In our experience, the $D$-optimality criterion will produce a design which favours time points with smaller response variance. Taking this into account simplifies the search.

- Obtaining the necessary information to construct $D$-optimum designs for experiments with cyclic blocks should not be a problem when several validation cycles can be carried out before the main study. In these cases, the optimality will still be approximately evaluated as the true model parameter values would change from setting to setting. However, the alternative (i.e., to randomise the order of the observations) is most likely to be considerably inferior.

- There are many other sequential experimental situations, not considered here, where our approach can still be very useful. However, if no validation study could be carried out, or information about the trend cannot be formulated, it is likely that randomising the order of the observations of the designs that are $D$-optimum in the absence of trend would provide a design that is more robust than a design that is obtained using arbitrary model parameter values in the design construction. On the other hand, experimentation has a sequential nature. For that reason the limitations of the approach described here, while real, are not very restrictive. 
Further complications may arise if the different response variables are not independent, when amending the design criterion accordingly may useful. Certainly, the methodology presented in this paper can also be described in terms of the approximate design theory. The method and how it can be used are described, for example, in Atkinson et al. (2007).

\section{References}

Atkinson, A. C. and R. D. Cook (1995). D-optimum designs for heteroscedastic linear models. Journal of the American Statistical Association 90, 204-212.

Atkinson, A. C. and A. N. Donev (1996). Experimental designs optimally balanced for trend. Technometrics 38, 333-341.

Atkinson, A. C., A. N. Donev, and R. Tobias (2007). Optimum Experimental Designs, with SAS. New York: Oxford University Press.

Cheng, C.-S. (1990). Construction of run orders of factorial designs. In S. Ghosh (Ed.), Statistical Design and Analysis of Industrial Experiments. New York: Dekker.

Cook, D. R. and L. Thibodeau (1980). Marginally restricted $d$-optimal designs. Journal of the American Statistical Association 75, 366-371.

Cox, D. R. (1951). Some systematic experimental designs. Biometrika 38, 312-323.

Donev, A. N. and A. C. Atkinson (1988). An adjustment algorithm for the construction of exact D-optimum experimental designs. Technometrics 30, 429-433.

Harman, R. and G. Sagnol (2015). Computing D-optimal experimental designs for estimating treatment contrasts under the presence of a nuisance time trend. In A. Steland, 
E. Rafajlowicz, and K. Szajowski (Eds.), Stochastic Models, Statistics and Their Applications, Volume 112. Springer Proceedings in Mathematics and Statistics.

Jones, B. and P. Goos (2011). Optimal Design of Experiments: a Case Study Approach. Hoboken, NJ, USA: John Wiley \& Sons, Inc.

Jones, B. and P. Goos (2015). Optimal design of blocked experiments in the presence of supplementary information about the blocks. Journal of Quality Technology 47, 301-317.

Nelder, J. A. and R. Wedderburn (1972). Generalized linear models. Journal of the Royal Statistical Society. Series A 135 (3), 370-384.

Pitman, E. J. G. (1936). Sufficient statistics and intrinsic accuracy. Mathematical Proceedings of the Cambridge Philosophical Society 32, 567-579.

Rosa, S. and R. Harman (2016). Optimal approximate designs for estimating treatment contrasts resistant to nuisance effects. R. Stat Papers 57, 1077-1106.

Stufken, J. and M. Yang (2012). Optimal Designs for Generalized Linear Models, in Design and Analysis of Experiments: Special Designs and Applications, Volume 3 (ed K. Hinkelmann). Hoboken, NJ, USA: John Wiley \& Sons, Inc.

Tack, L. and M. Vandebroek (2002). Trend-resistant and cost-efficient block designs with fixed or random block effects. Journal of Quality Technology 34, 422-436.

Tack, L. and M. Vandebroek (2003). Semiparametric exact optimal run orders. Journal of Quality Technology 35, 168-183. 
Tack, L. and M. Vandebroek (2004a). Budget constrained run orders in optimum design. Journal of Statistical Planning and Inference 124, 231-249.

Tack, L. and M. Vandebroek (2004b). Trend-resistant and cost-efficient cross-over designs for mixed models. Computational Statistics and Data Analysis 46, 721-746.

Wang, Y. and M. Ai (2016). Optimal designs for multiple treatments with unequal variances. Journal of Statistical Planning and Inference 171, 175-183. 


\section{Appendix.}

Table 2: Locally $D$-optimum design for the response $Y_{1}$.

\begin{tabular}{rrrrrr}
\hline Day & $x_{1}$ & $x_{2}$ & $x_{3}$ & $x_{4}$ & Time \\
\hline 1 & 1 & 1 & -1 & 0 & -1.0 \\
2 & 1 & -1 & -1 & 1 & -1.0 \\
3 & -1 & 1 & -1 & -1 & -1.0 \\
4 & 1 & -1 & 1 & -1 & -1.0 \\
5 & -1 & -1 & 1 & -1 & -1.0 \\
1 & 1 & 1 & -1 & -1 & -0.5 \\
2 & -1 & 1 & -1 & 1 & -0.5 \\
3 & -1 & -1 & -1 & 0 & -0.5 \\
4 & 1 & 1 & 1 & 1 & -0.5 \\
5 & 1 & 1 & 1 & -1 & -0.5 \\
1 & 1 & -1 & 1 & -1 & 0.0 \\
2 & 1 & -1 & 1 & 1 & 0.0 \\
3 & 1 & -1 & 1 & 1 & 0.0 \\
4 & -1 & -1 & 1 & 0 & 0.0 \\
5 & -1 & -1 & 1 & 1 & 0.0 \\
1 & -1 & 1 & -1 & 1 & 0.5 \\
2 & 0 & -1 & -1 & 0 & 0.5 \\
3 & -1 & -1 & -1 & 0 & 0.5 \\
4 & 1 & 1 & 1 & 0 & 0.5 \\
5 & -1 & -1 & 1 & 1 & 0.5 \\
1 & -1 & 1 & 1 & 1 & 1.0 \\
2 & 0 & 1 & 1 & 0 & 1.0 \\
3 & 1 & -1 & 0 & 1 & 1.0 \\
4 & -1 & -1 & -1 & 1 & 1.0 \\
5 & 1 & -1 & 1 & 0 & 1.0 \\
\hline
\end{tabular}


Table 3: Locally $D$-optimum design for the response $Y_{2}$.

\begin{tabular}{rrrrrr}
\hline Day & $x_{1}$ & $x_{2}$ & $x_{3}$ & $x_{4}$ & Time \\
\hline 1 & 1. & -1. & 1. & 1. & -1.0 \\
2 & 1. & 1. & 1. & 0. & -1.0 \\
3 & -1. & -1. & -1. & 1. & -1.0 \\
4 & -1. & 0. & -1. & -1. & -1.0 \\
5 & -1. & -1. & 1. & -1. & -1.0 \\
1 & -1. & 1. & -1. & 0. & -0.5 \\
2 & 1. & -1. & -1. & -1. & -0.5 \\
3 & -1. & 1. & 1. & 1. & -0.5 \\
4 & 1. & 1. & -1. & -1. & -0.5 \\
5 & 1. & 0. & -1. & 1. & -0.5 \\
1 & -1. & 1. & 1. & -1. & 0.0 \\
2 & -1. & 0. & 1. & 0. & 0.0 \\
3 & -1. & -1. & -1. & 1. & 0.0 \\
4 & 1. & 1. & -1. & 1. & 0.0 \\
5 & 1. & -1. & 1. & -1. & 0.0 \\
1 & 1. & 1. & 1. & 1. & 0.5 \\
2 & 1. & -1. & 1. & 1. & 0.5 \\
3 & -1. & -1. & -1. & -1. & 0.5 \\
4 & -1. & -1. & 1. & 0. & 0.5 \\
5 & 1. & 1. & -1. & -1. & 0.5 \\
1 & -1. & 1. & -1. & 1. & 1.0 \\
2 & 1. & -1. & -1. & 0. & 1.0 \\
3 & -1. & 0. & 1. & 1. & 1.0 \\
4 & -1. & 1. & 1. & -1. & 1.0 \\
5 & 1. & 0. & 1. & -1. & 1.0 \\
\hline & & & &
\end{tabular}




\section{Designing Experiments in Cycles}

\section{by Alexander N. Donev and Camilla Sammut-Powell}

\section{Highlights}

This paper identifies important problems in sequential experimental designs that have never been studied before, i.e. when:

- time trend in both the mean and the variance affects the results,

- time trend affects a response that is not normally distributed,

- time trend affects a multivariate response.

We propose a general methodology for designing such experiments, based on the framework of generalised linear models.

We also identify special types of experimental blocks, which we call cycles. As an example, we provide a typical industrial study where they occur naturally. We show how a locally D-optimum experimental design can be constructed in such a case and provide computer code that can be used to reproduce our results, or to construct similar experimental designs for different situations. The experimental designs constructed using the proposed methodology are not only robust to the possible time trend affecting the results, but also allow for incorporating useful available information about the study in the design construction. This makes the experiment where the designs will be used well focussed and the results more accurate and clearer than if conventional experimental designs are used instead.

As experimentation is sequential by nature, our results add an important contribution to the theory of experimental design. We believe the topic is very important to readers of the journals who are involved in experimental research. 